\title{
Digitalization of Russian University Educational Space
}

\author{
Ramil R. Khayrutdinov (a), Anastasia V. Fakhrutdinova* (b), Flera G. \\ Mukhametzianova (c), Irina V.Terenteva (d) \\ (a), (b), (c), (d) Kazan Federal University, 420008, Kazan (Russia), 18 Kremlyovskaya street, \\ avfach@mail.ru
}

\begin{abstract}
At present, education is not only a source of educational services but also the most important socio-economic institution, by means of which knowledge is transferred and a new level of civilization is formed. Each teacher at a modern university is faced with the phenomenon of digital technologies, which in turn are poorly subjected to external control. At the same time, search for indicators of educational institutions efficiency increase.

The problem is viewed in the context of understanding of the digital economy in terms of global leadership. This defined the purpose of the study, which is to find factors that influence the formation of the personality of a modern teacher - a teacher of history - in the process of professional training.

This study is based on analytically-synthetic and institutional theoretical approaches and comparative-generalizing theoretical method, as well as on following empirical methods: questionnaire analysis, included observation, interviews, focus groups study. Students of the History Faculty control and the experimental groups $59 \& 58$ were involved. The study also involved 35 teachers (aged 29 to 68) in 2018-2019 academic year.

As a result of the research, we found a number of risks that influence the formation of the personality of a modern teacher - a teacher of history. Digitalization of the university's educational space in the preparation of a future teacher leads to the disappearance of live communication and the transfer of implicit knowledge, changing the abilities for empathy, experience and complicity and, forming neurosis, depression, mistrust of others and balanced combination of collective and social-communicative forms of interaction with the active involvement of digital technologies is important.
\end{abstract}

Keywords: student, digitalization, university, educational space, future teacher, digital technologies.

(C) 2020 Ramil R. Khayrutdinov, Anastasia V. Fakhrutdinova, Flera G. Mukhametzianova, Irina

\section{V.Terenteva}

This is an open access article distributed under the terms of the Creative Commons Attribution License (CC BY 4.0), which permits unrestricted use, distribution, and reproduction in any medium, provided the original author and source are credited.

Published by Kazan Federal University and peer-reviewed under responsibility of IFTE-2020 (VI International Forum on Teacher Education)

\footnotetext{
* Corresponding author. E-mail: avfach@mail.ru
} 


\section{Introduction}

Digitalization of the Russian university educational space as a factor of success of students-future teachers is a result of a new technological era, which caused the blurring of boundaries between physical, biological and information educational technologies. Firs, personalities of trainees and student are no mare subjects of real social relations because now communication often occurs in a virtual social environment. Second, the era of development and implementation of digital technologies has led to systemic and functional changes in all areas of business, society, politics, and, in particular, university education. This results in the formation of a new University as a scientific and educational space which is viewed as a factor of student success in professional and personal development (Fakhrutdinova, 2015). At the same time, problems of digitalization in education in the context of challenges and risks for students remain unresolved. We tend to believe that problem of educational space design for future teachers is based on modern digital technologies and can be successfully solved in the context of scientific and technological progress, taking into account risks and challenges of the scientific and educational space for its participants.

\section{Purpose and objectives of the study}

The purpose of this work is to find the features of the formation of the personality of a modern history teacher in the process of professional training in a digital scientific and educational environment from the perspective of his or her personal and professional development.

\section{Literature review}

The research is based on the theory of Polterovich (1999) about the trajectory of the world institutional development and practice for solving modernization projects taking into account the features of civil culture and the mutual influence on economic growth as well as on the idea that education at all its levels should be considered not only as a market for educational services but also as an important socio-economic institution that transmits knowledge (explicit and implicit) and forms a new level of civilization in the era of mass digitalization, as Volchik and Maslyukova (2019) note. At the same time, the education system at all levels is not immune from so-called "dark ages", especially during the coronavirus pandemic, which has no borders and nationality (Nichols, 2017).

Every teacher of a modern university, especially during the pandemic and the mass transition to distance learning, has encountered the phenomenon of digital technologies related to the transfer of implicit knowledge, associated with the experience of the teacher, individual forms of knowledge to transfer and 
often associated with unclear and unfamiliar technologies (Fakhrutdinova \& Kondrateva, 2016). This in turn is difficult for internal and external control of students' and teachers' success. This phenomenon was previously pointed out in the works by Hutner, Krucken, \& Ninger (2018). In this respect, research indicates the effectiveness of educational institutions in terms of individual development when using digital technologies. Still, we have to mention that the effectiveness of the teaching staff often depends on the administrative resource. In higher education, there is a need to solve complex problems accumulated in the sphere of digitization related to the development level of the national and regional economy, social policy, and accounting understanding of the problem of human capital formation.

Digitalization is a new social digital environment, and the environment inevitably forms psychophysical changes of a person in accordance with the theory of Vernadsky (1998). Digitalization leads to the emergence of new types of knowledge and activities and digitizing traditional ones. This affects the education system in general and higher education in particular. In this respect, it is important to address the research to the process of training professionals, in our case, teacher training (Zhuk, 2018).

\section{Methodology}

Our research is based on an understanding of the digital economy and education as an economy of global leadership and global education. Global digitalization has defined the development of modern fundamental science and high-tech production, including intellectual production. The concept of the digital economy received international recognition in 2016 after the World Bank report "Digital dividends", in which this concept is considered as a system of economic, social and cultural relations based on digital information and communication technologies. In this broad definition, the concept of digital economy merges with the concept of digitalization. This is probably the bases of the phenomenon of digitalization or digital transformation of society.

This study was conducted to identify the positive effects of digitalization of the educational space and the problems that arise for both teachers and students, as well as to define the mechanisms for overcoming these problems. In our research, we consider digitalization as a change in the paradigm of thinking and activity of subjects of the scientific and educational space, mechanisms of communication with the external environment and with each other. Digital technologies in the subject-oriented educational space are the tool rather than the goal of the digital economy. 
This study relies on the following approaches:

- analytical and synthetic approach for analyzing published works on the research topic and determining mechanisms for assessing the challenges of the digital educational space in the educational process of future history teachers in the conditions of digitalization of Russian society and the risks of cognitive, axiological and humanistic, and personal-activity development of students;

- an institutional approach to identify and objectively assess institutional pitfalls in higher education when using digital technologies.

Methods used are:

- comparative and generalizing methods for comparing differences in the assessment of cognitive, axiological and humanistic, and personal-activity development of students and substantiating conclusions;

- empirical methods, including questionnaires proceeding and analysis, included observation, interviews, focus groups observation, and narrative analysis of the phenomenon of institutional traps in higher education.

117 students ( 3 and 4 years of study) of the Department "teacher of history and English" and "teacher of history and social studies" of the Faculty of history took part in the experiment. The control group (CG) had 59 students and the experimental group (EG) had 58 students. This study also involved 35 teachers (aged 29 to 68 years) from the Institute of International Relations of Kazan Federal University. The experiment was conducted during the 2018-2019 academic year. For students (EG), the learning process was built with the intensive involvement of information technologies. $50 \%$ of the training time was planned for work in the digital space. In our study of the realities and prospects of digitalization of the scientific and educational space in the educational environment of the University, from the position of increasing the success of future teachers in the context of personal and professional growth, we considered issues related to cognitive, axiological and humanistic, and personal-activity development. Cognitive development of the future teacher of history teacher implies the use of a large amount of information during the task preparation. This means the importance of understanding of the relevance of the information, the presence of good skills for conducting deep and reliable research, the ability to use a sufficient number of sources of information and new knowledge correctly, critically select and systematize the material. Axiological and humanistic development implies the development of socio-cultural responsibility, commitment to the truth, understanding of the value of social interaction, the responsibility 
to the historical accuracy of the material presented, and adherence to humanistic values. Personal - activity development implies responsibility and performance, conscientiousness, initiative, activity that consists in the successful use of variable search technologies, implementation of acquired knowledge and their extrapolation.

The process of digitalization of the educational space of students- future history teachers implies: using the results of digitization of the scientific and cultural and historical heritage of the world in the educational process (electronic libraries and museums, video, audio, interactive and digital publications, etc.); online events (online broadcasts, web conferences, distance learning and communication technologies, social networks, etc.); appeal to the technologies of socio-political, legal and economic interaction (egovernment, e-government, distance learning and communication technologies, social networks, etc.).

\section{Results}

The study was conducted at the Historical Department of the Institute of international relations of Kazan Federal University. And lasted for one academic year from September 2018, 117 third-and fourth-year students, who agreed to participate, were from four academic groups: two considered as experimental (EG) and two as control groups (CG). The purpose of the experiment was to determine the features of the formation of the personality of a modern teacher in the course of professional training in a digital educational environment.

At the initial stage, a study of cognitive, axiological and humanistic, personal-activity development was conducted. Since we studied the levels of professional and personal development, the tasks that allowed us to determine levels were related to the preparation of answers to a professional topic/task.

At the beginning of the academic year, 35 teachers involved in the educational process of experimental and control groups prepared tasks, the answers to which allowed to evaluate:

- axiological and humanistic development by the following parameters: 1) the culture of presentation of the material, 2) accuracy of the stile and data, 3) the social significance of the work performed, 4) cultural and historical accuracy, 5) compliance with accepted humanistic values of the society;

- cognitive search development according to the following parameters: 1) ability to use valid databases 2) use of a sufficient number of sources, 3) disclosure of the topic (from sufficient to deep), 4) ability to organize the material, 5) critical assessment of information; 
- personal and activity development according to the following parameters:1) completion of the work term, 2) seeking advice from a teacher, consultant if necessary, 3) completeness of the task (from sufficient to deep), 4) use of creative and variable research technologies, 5) desire and willingness to submit research materials.

Each position from all three blocs allowed them to get a maximum of 20 points, and the maximum number of points for the entire block was 100. Distribution of levels for each position: Low 0-5 points, Below average 5-10 points, Average/Medium 10,1-15 points, Above average 15,1-17 points, High 17,1-20 points. Distribution of levels for entire block : Low 0-25 points, Below average 25.1-50 points, Average/Medium 50,1-75 points, Above average 75,1-85 points, High 85,1-100 points.

At the end of the first semester, we conducted an interim interview and a survey where we asked students of both groups to identify problems related to their studies, as well as their own assessment of their condition. Teachers working with students of the experimental group were interviewed, too. After that, adjustments were made to the organization of work with digital databases for students from the experimental group.

In May 2019, at the end of the second semester of the academic year, we also conducted interviews with students (experimental group) and teachers aimed at identifying problems, opinions and suggestions related to the organization of the digital educational space.

After the experiment was completed, at the beginning of the term, the teachers of the experimental and control groups involved in the educational process prepared tasks that allowed them to assess the levels of axiological and humanistic, personal-activity and cognitive development of students in both groups.

The research has shown that today, there is an urgent need not only to declare arguments about the benefits and harms of digitalization for society and its subjects in general and for the education system in particular. The appearance of a new scientific, informational and social field is a phenomenon, which makes their use effective in case when people got used to it. It is important to mention that the modern education system implies a set of problems that are standard for society and at the same time, act as a stereotype of professional activity (Khovanskaya, Fakhrutdinova, \& Maklakova, 2017).

Unfortunately, a number of teachers, i.e. $10 \%$ of those participated in the experiment, still understand the digital modernization of education as tangible and point-based projects like Inograd, Skolkovo, Innopolis, and other centers of digitalization in different regions of Russia. In this context, modernization is reduced to technological aspects and attempts to imitate innovative technologies by involving the scientific elite 
into discussions, forums, meetups, symposiums, discussion panels, etc., subjecting the institutions of civilization to unfounded criticism, as Gontmakher (2010) mentions in his research.

During the discussion of the possibility of creating a digital educational environment for students of the history Department with teaching stuff, we faced the risks of organizing a digital subject-oriented scientific and educational space in the university environment, which we attributed to subjective factors, manifested 1) in the unreadiness of the teaching staff of the university to attract modern digital technologies in the educational process (12\% of teachers are all over 45 years old); 2) in the wrong understanding of intensification of education by digitalization and the following the loss of the subject-oriented attitude at the educational process, $24 \%$ of teachers - under 35 years of age); 3 ) in the absence of technical readiness, $10 \%$ of students and $6 \%$ of teachers (all over 45 years of age) (Namely, the lack of material and technical base, insufficient knowledge of digital literacy skills). At the same time, our research suggests that these risks can be overcome when taken into account and their understanding may become a significant factor in the success of training future teachers.

We have developed a program and recommendations for the introduction of modern digital technologies in the educational process after overcoming the difficulties associated with a poor understanding of the digitalization process urgency, the lack of material and technical base. Students were given access to laboratories, as well as classes for advanced digital literacy. Teaching staff on their own initiative took advanced training courses related to IT technologies. We have started to study the impact of digitalization of the scientific and educational space on the personal and professional development of students.

At the initial stage of the experiment, students demonstrated average and above-average levels of axiological and humanistic, cognitive development, and personal-activity aspects. The measurement of axiological and humanistic development showed that students have: 1) skills of presentation culture, adherence to humanistic values at a higher average level (according to these positions, they scored an average of 12, 5 points); 2) accuracy of the stile and data, cultural and historical accuracy in the performance of work in both groups of students were demonstrated at an average level (9.5 points); 3 )the social significance of the work performed was highly evaluated (16-18 points) $20 \%$ of students (CG) and (16-17 points) $22 \%$ (EG), while the majority demonstrated an average level but the average score was 9.5(CG) and 9 (EG); 4) cultural and historical accuracy was demonstrated at an average level of 10 (CG) and 9.5 (EG); 5) at the performance of humanistic values they scored an average of 15.5 points (CG) and a high level of 17 points (EG). 
Cognitive development demonstrated by the students, showed: 1) a low level of understanding of the validity of databases in more than $50 \%$ of students, but the average result obtained showed a level below average 7.5(CG) and 8 points(EG); 2) students sought to use the required minimum of number of sources, the average score obtained for this parameter is 6 , the level is below average(CG \& EG); 3) the topic was revealed at an average level of $12(\mathrm{CG})$ and 13 points(EG), which is due to the lack of understanding of the validity of databases and the limited sources used; 4) students demonstrated the ability to systematize material at a level close to the average-14(CG), 13.5 points(EG); 5) critical assessment of information 55\% of students(CG) and 50\%(EG) at a level above average, the remaining students at the average level had an average score of -12.5 points in both groups.

Parameters of personal activity development showed the following data: 1) two students of the EG and one student of the CG passed the work in advance, while one student took the work for revision (EG). $70 \%$ of all students passed their work on time, but $30 \%$ of all students passed their work with a delay (in this case, the distribution of points between students of both groups is equal), the average number of points scored for this position -10 points, which is the average level of completion of work on time; 2) $10 \%$ of students in the experimental group and $15 \%$ of students in the control group came for consultation to professors, receiving higher scores, however, for this position, both groups showed a borderline level between low and below average, the average number of points for each group - 5; 3) the assessment of the task in terms of depth of analysis (from sufficient to deep) was demonstrated at an average level -8 points (CG) and 9 points $(E G), 4)$ the use of creative and variable research technologies is characterized by an average level 9.5 (CG) and 9 points(EG), 5) only $40 \%$ of the participants in the control group showed a desire and readiness to present their research materials 8 points out of $50 \%$ of the participants in the experimental group - 10 points (Table 1).

Minor differences in the development of all three studied components (blocks), demonstrated by students of the control and experimental groups, allowed us to consider the study legitimate (Table1).

Table 1. Distribution of levels \& points before the experiment in EG and CG

\begin{tabular}{ccccc}
\hline Development & \multicolumn{3}{c}{ CG } & \multicolumn{2}{c}{ EG } \\
\cline { 2 - 5 } & Score & Level & Score & Level \\
\hline Axiological \& humanistic & 57 & Average & 57,5 & Average \\
Cognitive & 52 & Average & 53 & Average \\
Personal activity & 40,5 & Below average & 43 & Below average
\end{tabular}

Conducted in the middle of the experiment, December 2018, surveys and interviews of students (control and experimental groups) and teachers allowed us to identify a number of problems faced by the 
participants of the experiment. Among the difficulties encountered by students, there was increased fatigue, and a decline in interest in the educational process. We have suggested that one of the reasons could be the lack of social alive interaction. Another reason mentioned by the participants of the experiment was that most of the work was performed remotely, and students had to work with a large number of different databases, while they had difficulties in identifying significant materials and valuable databases themselves. To solve this problem, teachers were recommended to insist on regular consultation for students when preparing tasks. We also strengthen the social significance of the educational process in accordance to numerous research data from the laboratory of Andrey Kurpatov, according to which digitalization and digital technologies do not always have a positive impact on the human brain, and this leads to the loss of initiative and depression, a person needs live communication and time free from "gadgets". In this regard, the educational process was revised, more attention was paid to group tasks, and the scope of presentation of completed tasks was also expanded. In other words, the social, creative, and creative components were added (Kurpatov, 2019).

The involvement of the digital environment in the educational process allowed to increase its intensification, but the results became more significant in the second half of the experiment after the creative social and creative components were involved.

Results of measurements obtained in May 2019, showed differences in the measurements in the control and experimental groups, that allows to say about the positive impact of the digital scientific and educational environment from a position of personal and professional development of future teachers in the training process.

The measurement of axiological and humanistic development showed that students developed: 1) cultural skills of presentation of material, 5) commitment to humanistic values to above average and high, according to these positions, they scored an average -15 points (CG) and 17.5 points (EG); 2) accuracy and accuracy of data, 4) cultural and historical accuracy of the work of students in both groups were demonstrated at the average level and above average - 12 (CG) and 15 points (EG); 3) the social significance of the work performed received an average level- 12.5 points (CG) and a high level -18 points (EG).

Cognitive development demonstrated by students showed 1) almost an average level of understanding of database validity -9 points (CG) and high level -16 points (EG);2) students in the control group sought to use the medium specified number of sources average level obtained for this parameter at the bottom -11 points - average(medium) level and 16 points among students in the experimental group, 3) disclosure of 
the topic was demonstrated between average and above-average - 15 (CG) and high level 17 points (EG), due to insufficiently developed level of understanding of the validity of the databases, participants of the control group; 4) students demonstrated the ability to systematize material at an above-average level-15 (CG) and at a high level - 17 points(EG); 5) they demonstrated the ability to evaluate information critically - 14 points(CG) and 17 points (EG) -above average and high levels.

Parameters of personal activity development showed the following data: 1) students of the experimental group passed all the work before the deadline because during the year they performed group projects in which an individual block, not completed in time could cause problems for other group members. This contributed to the development of responsibility. $98 \%$ of the works were completed in advance, one work was submitted with a delay, which was due to personal difficulties that occurred at one student and one work was submitted almost at the deadline; thus, the average score of the experimental group is 19 points high level, while the control group has an average of 12 points. 2) attitude of the students for teaching stuff advice: 15.5 points - high-level(EG), while the control group demonstrated a medium level - 9 points; 3 ) evaluation of the job, in terms of depth of analysis was demonstrated at an average level 10 points (CG) and high level - 15 points (EG); 4) the use of creative and divergent technologies for research is characterized by below-average level 9.5 points (CG) and average level- 15 points (EG); 5) only 35\% of the control group participants showed a desire and readiness to present their research materials -7 points out of $90 \%$ of the experimental group participants - 18 points (Table 2 ).

Table 2. Distribution of levels and points after the experiment in EG and CG

\begin{tabular}{ccccc}
\hline Development & \multicolumn{3}{c}{ CG } & \multicolumn{2}{c}{ EG } \\
\cline { 2 - 5 } & Score & Level & Score & Level \\
\hline Axiological \& humanistic & 66,5 & Average & 83 & Above average \\
Cognitive & 64 & Average & 83 & Above average \\
Personal activity & 47,5 & Below average & 82 & Average
\end{tabular}

Thus, we have demonstrated the positive aspects of digitalization of the educational space, difficulties that arise, both for teachers and students, and identified mechanisms, and ways to overcome them.

The study showed that there is an urgent need to move from declaring arguments about the benefits and harms of digitalization for society and its subjects in general and for the education system in particular towards a new scientific, informational and social field and the search for mechanisms for the objective study of this phenomenon, which will make its use less socially traumatic and more effective. 


\section{Discussions}

Today, Russian universities form an educational environment and scientific space in which not only explicit but also implicit knowledge is created, this influences the quality and quantity of various educational programs developed on the basis of social values using new digital educational technologies (Mukhametzyanova \& Miftakhov, 2013; Volchik \& Maslyukova, 2019).

Digital technologies in higher education and specific transformation of universities lead to improvement of the educational process (Kozlova, 2019).

The experiment allows to consider that the problems of using innovations in higher education through the prism of psychological and organizational aspects of introducing digital education may help to avoid its transformation into a" digital collective farm", i.e. falling into the trap of digitalization (Konrateva \& Fakhrutdinova, 2017; Kramarenko, 2017). Thus, at the end of the experiment, $78 \%$ of students and $94 \%$ of teachers who participated in the experiment noted that creativity used in the educational process contributes to the development of both cognitive and social skills of students. The correlation of questions about the study, development, and formation of giftedness and creativity in subjects of educational activity, especially on the basis of digitalization technologies (Morozov, 2019; Khayrutdinov, Mukhametzyanova, Venidiktova, Chugunov, \& Morozov, 2019).

\section{Conclusion}

The use of digital technologies in higher education has a generally positive impact, i.e. the possibility of actualization, intensification, and individualization of the educational process, as well as negative, i.e. hidden from the outside observer's influence.

The procedural component of the project showed that the main risk of organizing of a digital subjectoriented educational space has some subjective factors, namely, the unreadiness of the teaching staff of the university to attract modern digital opportunities of the global space in the educational process, and wrong understanding of intensification due to digitalization through the loss of the subject-oriented orientation of the educational process due to the attraction of new technologies (Mukhametzyanova, Yarullina, Vafina, \& Biserova, 2012). At the same time, our research suggests that the risks can be overcome and the digitalization of the university educational space, taking into account the risks and sufficient knowledge of the mechanisms for overcoming them, is a significant factor in the future professional success of students, future teachers. Digitalization of the educational process may lead to the disappearance of live communication and the transfer of implicit knowledge, changing the ability to empathy, experience, and 
participation, and forming neuroses, depression, and distrust of others. In order not to change the subjectivity of the individual and not to lose interest in self-realization and self-actualization, it is important to use a balanced combination of creative and socio-communicative forms of interaction with the active involvement of digital technologies. Throughout the entire development of mankind, the word was understood as a psychological tool of development.

\section{Acknowledgements}

We are grateful to Kazan Federal University for providing the opportunity to prepare an article and conduct research.

\section{References}

Fakhrutdinova, A. V. (2015). University educational process development - modern understanding and principles of scientific research. Kazan State Academy of Veterinary medicine, 4(224), 233-236.

Fakhrutdinova, A. V., \& Kondrateva, I. G. (2016). Contemporary tendencies of social tutoring in period of multiculturalism: moral characteristic. Man in India, 96(3), 853-858.

Gontmakher, E. (2010). Russian Modernization: Institutional Traps and Civilization Landmarks. International Economics and International Relations, 10, 3-11.

Hutner, O., Krucken, G., \& Ninger, G. (2018). Education Institutions as Organizations. Developments in International Perspective. Cham: Springer.

Kozlova, N. S. (2019). Digital technologies in education. Bulletin of Maykop State Technological University, 1(40), 85-93.

Konrateva, I. G., \& Fakhrutdinova, A. V. (2017). Questions of continuous education. Foreign languages in modern world. Proceedings of X International conference, 140-147.

Kramarenko, N. S. (2017). Psychological and organizational aspects of the introduction of digital education, or how to introduce innovations cannot be turned into a "digital collective farm". Bulletin of Moscow State Regional University, 4, 1-16.

Kurpatov, A. (2019). New reality of digital civilization. Retrieved from https://www.youtube.com/watch?v=ePK82v0d6W8 
Khayrutdinov, R. R., Mukhametzyanova, F. G., Venidiktova, E. A., Chugunov, A. S., Morozov, A. V. (2019). Historical significance of the image of the Kazan virgin in the education of spirituality, morality, and patriotism. Humanities and Social Sciences Reviews, 7(5), 682-686.

Khovanskaya, E. S., Fakhrutdinova, A. V., \& Maklakova, N. V. (2017). University as a social phenomenon. Man in India, 97(9), 101-107.

Morozov, A. V. (2019). To the question of the correlation of the concepts of "giftedness" and "creativity" in modern psychology. Personality in space and time, 8, 136-145.

Mukhametzyanova, F. G., \& Miftakhov, I. I. (2013). The phenomenon of student subjectivity in psychology. Kazan Pedagogical Journal, 4(99), 126-130.

Mukhametzyanova, F. G., Yarullina, A. S., Vafina, V. R., \& Biserova, G. K. (2012). University student as a subject of educational and professional activity. Kazan Pedagogical Journal, 5-6 (95), 20-27.

Nichols, T. M. (2017). The death of expertise: the campaign against established knowledge and why it matters. London: Oxford University Press.

Polterovich, V. M. (1999). Institutional traps and economic reforms. Economics and Mathematical Methods, 35(2), 3-20.

Vernadsky, V. I. (1998). The biosphere. London: Springer Science \& Business Media.

Volchik, V. V., \& Maslyukova, E. V. (2019). Reforms, sloppy knowledge and institutional traps in education. Terra Economicus, 17(2), 146-162.

Zhuk, A. A. (2018). Institutional traps in higher education. Actual problems of economics and law, 12(4), 789-799. 